\title{
Effect of Substituent Groups on Hydrogen Bonding of Polyhydroxystyrene Derivatives
}

\author{
Kunio Nakamura, Tatsuko Hatakeyama, ${ }^{*}$ and Hyoe Hatakeyama** \\ Industrial Research Institute of Kanagawa Prefecture, \\ 3173, Showamachi, Kanagawa-ku, Yokohama 236, Japan \\ * Research Institute for Polymers and Textiles and \\ **Industrial Products Research Institute, \\ 1-1-4, Yatabe-Higashi, Tsukuba, Ibaraki 305, Japan
}

(Received November 19, 1982)

\begin{abstract}
Poly(2-hydroxystyrene) (PoHS), poly(3-hydroxystyrene) (PmHS), poly(4-hydroxystyrene) (PpHS), and their acetylated polymers were prepared. Attention was directed to the effect of substituent groups and sorbed water on the molecular motion of the polyhydroxystyrene derivatives. The glass transition temperature $\left(T_{\mathrm{g}}\right)$ of these polymers and the phase transition of water sorbed on the polymers were studied by differential scanning calorimetry (DSC). Bound water content was calculated from total amounts of sorbed water and crystallization enthalpy of water. The $T_{\mathrm{g}}$ of each polymer increases linearly with increasing mole fraction of hydroxystyrene (HS). It was clarified that the $T_{\mathrm{g}}$ increase is dependent on the replacement of acetoxyl groups with hydroxyl groups, since formation of hydrogen bonding occurs through the hydroxyl groups of the polymers. It was also found that the hydroxyl group of PmHS located at the 3-position of each aromatic ring formed hydrogen bonds the most readily, while that located at the 4-position did so second to this. The $\mathrm{OH}$ group of PoHS located at the 2-position formed hydrogen bonds with difficulty. Two exothermic peaks for the crystallization of water sorbed on each of the above polymers were observed on a DSC curve: one was a sharp peak (Peak I) of free water observed at about $255 \mathrm{~K}$, and the other, a broad small peak (Peak II) of freezing bound water observed at about $230 \mathrm{~K}$. Judging from the amounts of water calculated from the crystallization enthalpy of water sorbed on each polymer, there seems to be non-freezing water which does not crystallize. The bound water content of each polymer increased with increasing mole fraction of HS. The increase in bound water content of PmHS was highest with increasing mole fraction of HS.

KEY WORDS Polyhydroxystyrene / Substituent / Hydrogen Bond / Water / Bound Water / Glass Transition / Differential Scanning Calorimetry /
\end{abstract}

The hydroxyl groups of polyhydroxystyrene derivatives previously reported ${ }^{1-7}$ were located at the 4-position in the aromatic ring. The location of the hydroxyl group is believed to be important when discussing the relationship between $T_{\mathrm{g}}$ and the number of hydrogen bonds. Barb ${ }^{8}$ reported that the softening temperature of polystyrene derivatives decreased when hydrophobic group, such as the methyl group, was introduced onto the 3-position. These findings indicate that the molecular motion of polymers is markedly influenced by the location of substituent groups in the aromatic ring of polystyrene derivatives.

In the present study, a series of poly(hydroxystyrene) derivatives having hydroxyl group at the
2-, 3-, or 4-position of the aromatic ring were prepared by the hydrolysis of poly(2-acetoxystyrene) (PoAS), poly(3-acetoxystyrene) (PmAS), and poly(4-acetoxystyrene) (PpAS). The degree of hydrolysis was successively changed to obtain samples containing various amounts of hydroxyl groups. The effects of hydrogen bonding on these polymers were investigated by measuring $T_{\mathrm{g}}$ in the dry and humid states.

\section{EXPERIMENTAL}

\section{Sample Preparation}

Poly(2-hydroxystyrene) and poly(3-hydroxystyrene) were synthesized by a procedure similar 

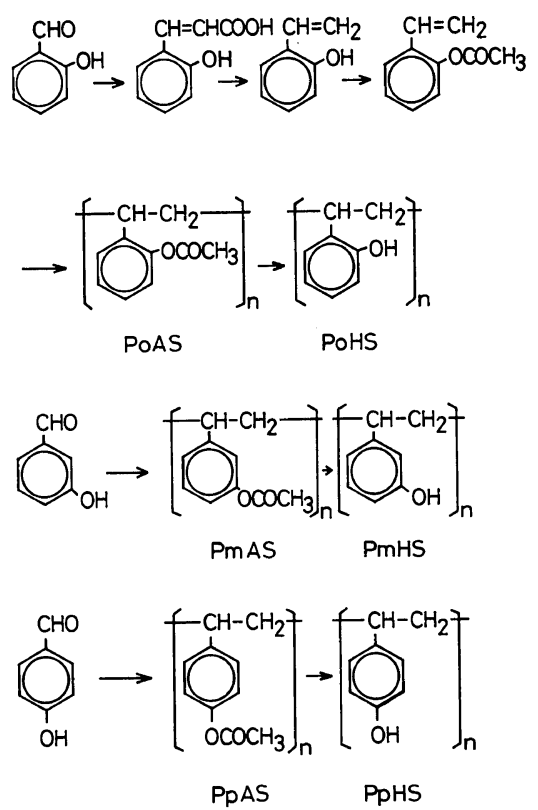

Figure 1. Scheme for PoHS and PpHS synthesis.

Table I. Molecular weight $\left(M_{w}\right)$, molecular weight distribution $\left(M_{w} / M_{n}\right)$ and glass transition temperature $\left(T_{\mathrm{g}}\right)$ of the polymers

\begin{tabular}{lccc}
\hline & $M_{w}$ & $M_{w} / M_{n}$ & $\frac{T_{\mathrm{g}}}{\mathrm{K}}$ \\
& & & 401 \\
PoAS & $6.0 \times 10^{4}$ & 2.7 & 445 \\
PoHS & $5.9 \times 10^{4}$ & 3.0 & 345 \\
PmAS & $3.6 \times 10^{4}$ & 2.9 & 440 \\
PmHS & $3.0 \times 10^{4}$ & 3.1 & 386 \\
PpAS & $3.6 \times 10^{4}$ & 3.5 & 455 \\
PpHS & $3.1 \times 10^{4}$ & 3.7 & 365 \\
PSt & $1.0 \times 10^{5}$ & 2.9 & \\
\hline
\end{tabular}

to that for obtaining poly(4-hydroxystyrene). ${ }^{1}$

In order to obtain partially hydrolyzed samples of PoAS, PoAS was dissolved in $500 \mathrm{ml}$ of acetone containing hydrochloric acid $(10 \mathrm{ml})$. Then the mixture was allowed to stand for various intervals of time $(1-24 \mathrm{~h})$ at $65^{\circ} \mathrm{C}$. The resulting mixture was poured slowly into a large excess of water (3 liters), filtered, repeatedly washed with water, and then dried in air. A similar process was applied to both PmAS and PpAS (Figure 1).

Polystyrene (PSt) was commercially obtained from the Pressure Chemical Company.

\section{Measurements}

Gel Permeation Chromatography (GPC). The molecular weight $\left(M_{w}\right)$ and molecular weight distribution $\left(M_{w} / M_{n}\right)$ of the polymers were measured on a Toyosoda gel permeation chromatograph, model HLC-801. Mono-disperse polystyrenes (PSt) (Pressure Chemical Co.) were used as calibration materials. The $M_{w}$ and $M_{w} / M_{n}$ of each polymer are listed in Table I.

Infrared Spectroscopy (IR). The degree of hydrolysis was measured with an infrared spectrophotometer, model IRG (Nippon Bunko Co. Ltd.). The degree of hydrolysis was calculated by the same method reported previously. ${ }^{2}$ The degree of hydrolysis of the polymer is shown by the mole fraction of hydroxystyrene (HS).

Differential Scanning Calorimetry (DSC). The glass transition temperature $\left(T_{\mathrm{g}}\right)$ of polymers and the phase transition phenomenon of sorbed water were studied by a Perkin-Elmer, differential scanning calorimeter DSC-II. The DSC curves were obtained at a scanning rate of $10^{\circ} \mathrm{C} \mathrm{min}^{-1}$. The melting and crystallization enthalpies were calculated by reference to the enthalpy of pure water.

Samples were sealed in volatile-sample aluminium pans previously exposed to steam in an autoclave at $120^{\circ} \mathrm{C}$ for $3 \mathrm{~h}$ to eliminate the formation of aluminium hydroxide during measurements.

The samples were first dried in a vacuum desiccator for about one week and then each $5 \mathrm{mg}$ sample was weighed. For measurement of polymers containing sorbed water, a small amount of water was added by a microsyringe and the total weight was then measured on a microbalance. If the water content exceeded the predetermined amount, the sample pan was allowed to stand for a few minutes until the water evaporated. The water content (water content $=$ weight of water/weight of dry sample $\times 100 \%$ ) was calculated by measuring the total weight after the sample pan had been sealed. Following the DSC measurement, the sample pan was again weighed so as to confirm that no weight loss had taken place during the measurement.

\section{RESULTS AND DISCUSSION}

Figure 2 shows the DSC curves of PoAS, PmAS, PpAS, PoHS, PmHS, PpHS, and PSt in the tem- 


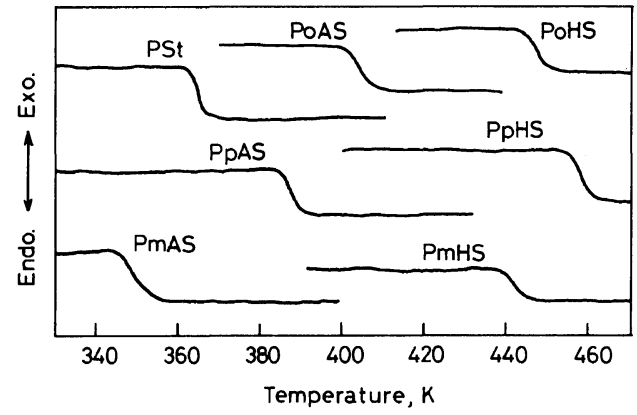

Figure 2. DSC curves showing $T_{\mathrm{g}}$ 's of PoHS, PoAS, PmHS, PmAS, PpHS, PpAS, and PSt.

perature region of glass transition. The glass transition temperature $\left(T_{\mathrm{g}}\right)$ values of these polymers are listed in Table. I. The $T_{\mathrm{g}}$ 's of the acetylated polymers are lower than those hydrolyzed. The acetoxyl group attached to the aromatic ring seemed to increase the intermolecular distance and the flexibility of the molecular chain. The $T_{\mathrm{g}}$ of PmAS is lower than that of PSt, indicating that the substituent group at the 3-position of the aromatic ring affects markedly the molecular motion of the main chain. The high $T_{\mathrm{g}}$ of PoAS $(401 \mathrm{~K})$ suggests that the acetoxyl group at the 2-position prevents the motion of the main chain. In the case of PoHS, PmHS, and PpHS, the hydroxyl groups attached to the aromatic rings seem to induce appreciable interaction between molecular chains, and depress the molecular motion of the main chain. Therefore, the $T_{\mathrm{g}}$ 's of PoHS, PmHS, and PpHS are higher than those of PoAS, PmAS, and PpAS.

Figure 3 shows the relationship between the mole fraction of hydroxystyrene (HS) and the $T_{\mathrm{g}}$ 's of PoHS, PmHS, and PpHS. The $T_{\mathrm{g}}$ of each polymer increases almost linearly with increasing mole fraction of HS. The $T_{\mathrm{g}}$ of PmAS was the lowest among the polymers. This indicates that the substituent group at the 3-position in the aromatic ring markedly enhances the molecular motion of the main chain. The $T_{\mathrm{g}}$ of PoAS is higher than those of PmAS and PpAS. The steric hindrance of the substituent groups at the 2-position is considered to prevent the main chain motion of the polymer. The $T_{\mathrm{g}}$ values increased $95 \mathrm{~K}$ in PmHS, $69 \mathrm{~K}$ in $\mathrm{PpHS}$, and $44 \mathrm{~K}$ PoHS as a result of the entire conversion of the acetoxyl groups to hydroxyl groups. Thus, it may be concluded that the $T_{g}$ in-

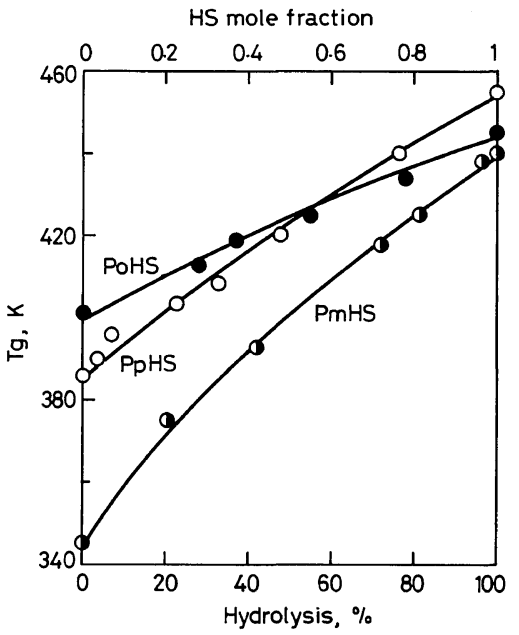

Figure 3. Relationship between mole fraction of HS and the $T_{\mathrm{g}}$ 's of variously hydrolyzed PoAS, PmAS, and PpAS.

creases, as a result of hydrogen bonding formation between the hydroxyl groups of the polymer chains. Moreover, hydrogen bonding occurs most readily between hydroxyl groups located at the 3position, and with difficulty at the 2-position.

We reported in the previous papers ${ }^{2-4}$ the relationship between sorbed water and molecular motion of polyhydroxystyrene derivatives. It was shown that the $T_{\mathrm{g}}$ of polyhydroxystyrene derivatives decreases with increasing amount of sorbed water. The "critical water content," obtained by calculating the necessary water content for decreasing the $T_{\mathrm{g}}$ 's of polyhydroxystyrene derivatives to that of polystyrene (PSt), could be used to quantify the number of hydrogen bonds between polymer chains. It was also clarified that the critical water content was almost equal to the "bound water content" estimated from the enthalpies of the phase transition of sorbed water detected by DSC. Therefore, it is reasonable to suggest that the number of hydrogen bonds in each of PoHS, PmHS, and PpHS can be estimated by measuring the bound water content.

As shown in Figure 4, when the water content of PoHS, PmHS, and PpHS exceeds a certain amount, two crystallization peaks are observed by DSC, as a sample is cooled from room temperature to about $200 \mathrm{~K}$ : a sharp crystallization peak (Peak I) at about $255 \mathrm{~K}$ and a broad peak (Peak II) at about $235 \mathrm{~K}$. In the crystallization of pure water, a sharp peak was 


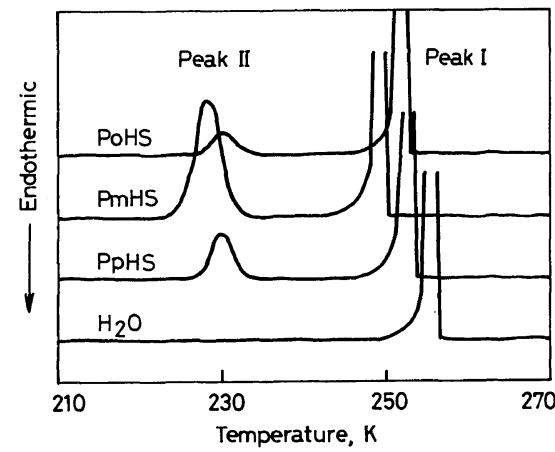

Figure 4. DSC cooling curves of pure water and water sorbed on PoHS, PmHS, and PpHS.

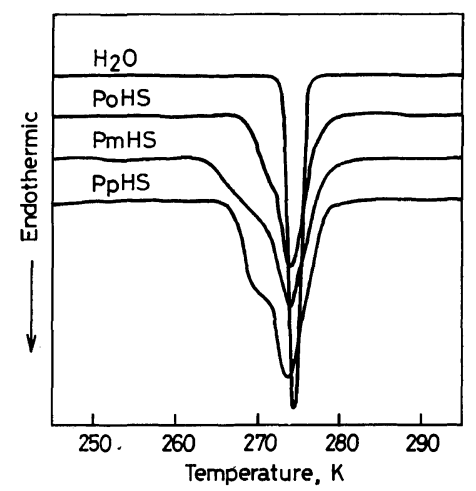

Figure 5. DSC heating curves of pure water and water sorbed on PoHS, PmHS, and PpHS.

observed at about $255 \mathrm{~K}$. Therefore, Peak I, found in the DSC crystallization curves of sorbed water in each polymer, should correspond to the crystallization of free water which is essentially the same as that of pure water.

By comparing the amount of added water with that calculated from the crystallization enthalpy, it was found that the calculated amount of water was smaller than that added. This suggests that there exists water which does not crystallize in the temperature range of our experiment $(200 \mathrm{~K})$. This water was designated as "non-freezing water," in which the first order phase transition is not observed in the temperature range. Peak II was a kind of bound water designated as "freezable bound water." Therefore, we categorized water adsorbed on the polymers as three kinds: free water (Peak I), freezable bound water (Peak II), and non-freezing bound water.

Figure 5 shows the melting curves of water sorbed

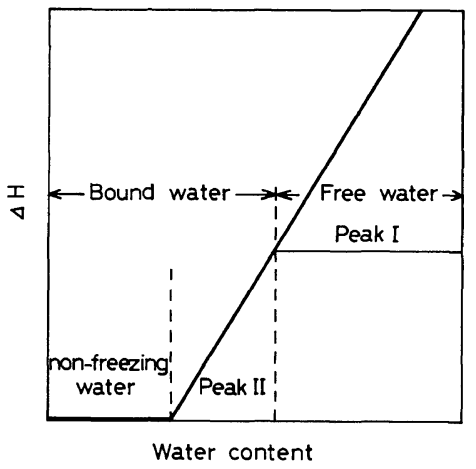

Figure 6. Schematic relationship between water content and crystallization enthalpy of water sorbed on polyhydroxystyrene.

for the polymers studied. These curves show a broad endothermic peak from about $265 \mathrm{~K}$ to $280 \mathrm{~K}$, which differs from the sharp peak for pure water. It should be noted that the melting peaks of two kinds of water merge into one another. If this melting curve can be divided into two peaks, the melting enthalpy equivalent to Peak I and Peak II can be obtained. However, an exact separation of the melting curve is difficult since no asymmetric pattern is suitable for adjusting it to Gaussian, Lorentzian or other distributional functions. Therefore, the quantity of "bound water" evaluated from the crystallization curve of sorbed water was used.

Figure 6 shows the schematic relationship between the amount of sorbed water and crystallization enthalpy. When the amount of sorbed water is small, there exists "nonfreezing water," in which no phase transition can be observed; when the amount of sorbed water slightly increases, Peak II is observed at a temperature lower than $235 \mathrm{~K}$. The area of Peak II increases as the water content increases and levels off at a certain amount of water which is specific to the kind to specimen. The "bound water" is defined as the summation of the non-freezing water and the water corresponding to Peak II. When the amount of water exceeds this value, a new crystallization peak (Peak I) appears at about $255 \mathrm{~K}$. The area of Peak I increases as the amount of sorbed water increases.

The amount of bound water as a function of the mole fraction of HS is presented in Table II. The maximum values of the bound water of PoHS, PmHS, and PpHS were $8.4 \%, 12.7 \%$, and $10.3 \%$, 
Table II. Mole fraction of HS and bound water content of each polymer

\begin{tabular}{clc}
\hline Polymer & $\begin{array}{c}\text { Mole fraction } \\
\text { of HS }\end{array}$ & $\begin{array}{c}\text { Bound water } \\
(\%)\end{array}$ \\
\hline \multirow{2}{*}{ PoAS } & 0 & 2.8 \\
& 0.35 & 3.8 \\
& 0.62 & 4.8 \\
PoHS & 0.78 & 6.7 \\
PmAS & 1 & 8.4 \\
& 0 & 3.8 \\
& 0.27 & 5.6 \\
PmHS & 0.41 & 6.8 \\
\hline \multirow{2}{*}{ PpAS } & 0.80 & 10.5 \\
& 1 & 12.7 \\
& 0 & 3.2 \\
& 0.09 & 3.6 \\
& 0.22 & 4.2 \\
PpHS & 0.37 & 4.8 \\
& 0.43 & 5.6 \\
& 0.79 & 8.0 \\
& 1 & 10.3 \\
\hline
\end{tabular}

respectively. In previous reports, it was clarified that the $T_{\mathrm{g}}$ 's of polymers with hydroxyl groups increase as the numer of hydrogen bonds increases. At the same time, water molecules sorbed in the polymer breaking the hydrogen bonds between the hydroxyl groups and the water molecules become tightly attached to the hydroxyl groups of the polymers. As shown in the table, the relationship between the location of the hydroxyl groups in the aromatic ring and the bound water content suggests that having a hydroxyl group at the 3-position forms hydrogen bonds most readily. In contrast, it is more difficult for PoHS with its hydroxyl group at the 2-position in the aromatic ring, to make hydrogen bonds because of the steric hindrance between the main chain and the hydroxyl group. As shown in Table I, however, the order of the $T_{\mathrm{g}}$ 's of polyhysroxystyrene derivatives is PpHS $>$ PoHS $>$ PmHS. PmHS, which forms hydrogen bonding the most readily, has the lowest $T_{\mathrm{g}}$. It is quite probable that the location of $\mathrm{OH}$ groups at the 3-position of aromatic rings, is responsible for this rate of formation and breaking up of hydrogen bonding. However, it is difficult to provide more conclusive evidence for this based on the data obtained by thermal analysis.

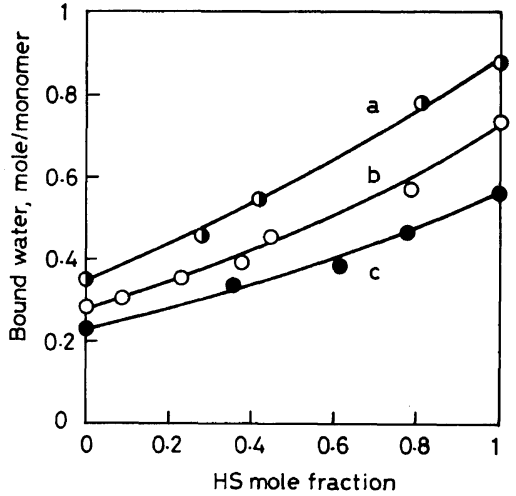

Figure 7. Relationship between mole fractions of HS and amount of water sorbed on each monomer unit of variously hydrolyzed PoAS, PmAS, and PpAS. a, PmHS; b, PpHS; c, PoHS.

Figure 7 shows the amount of water sorbed by monomer unit of each polymer calculated from the amount of bound water. As mentioned above, it is reasonable to consider that water molecules first break up the hydrogen bonding between polymer chains and then form new hydrogen bonds between hydroxyl groups of the polymers and water molecules. Therefore the amount of water sorbed per monomer unit of each polymer can be regarded as an index of the polymer's ability for hydrogen formation. The number of water molecules attached to the monomer unit of each polymer increase with increasing mole fraction of HS, as shown in Figure 7. The amount of water sorbed on PoHS, PmHS and PpHS was $0.56,0.85$, and $0.69 \mathrm{~mol}$, respectively. It is recognized that these three polymers differ in their ability to form hydrogen bonding.

It may be concluded that the location of hydroxyl groups markedly affects the readiness with which hydrogen bonds are formed between polymers. This was confirmed by vaiations in $T_{\mathrm{g}}$ and bound water content. The effect of the location of each hydroxyl group at the aromatic ring on the readiness of the formation of hydrogen bonding is in the order of the 3->4->2-position.

Acknowledgment. The authors are grateful to Professor Ronald O. Linden, University of Library and Information Science, for his kind assistance in preparing this munuscript. 


\section{REFERENCES}

1. T. Hatakeyama, K. Nakamura, and H. Hatakeyama, Polymer, 19, 593 (1978).

2. K. Nakamura, T. Hatakeyama, and H. Hatakeyama, Polymer, 22, 473 (1981).

3. K. Nakamura, T. Hatakeyama, and H. Hatakeyama, Kobunshi Ronbunshu, 38, 763 (1981).

4. K. Nakamura, T. Hatakeyama, and H. Hatakeyama,
Kobunshi Ronbunshu, 39, 53 (1982).

5. H. Hatakeyama, K. Nakamura, and T. Hatakeyama, Pulp and Paper Mag. Canada, 6, TR-105 (1980).

6. T. Hatakeyama and H. Hatakeyama, Polymer, 23, 475 (1982).

7. T. Hatakeyama, K. Nakamura, and H. Hatakeyama, Netsusokutei, 6, 50 (1979).

8. W. G. Barb, J. Polym. Sci., 37, 515 (1959). 